\title{
A case of macular subretinal neovascularisation in chronic uveitis probably caused by sarcoidosis
}

\author{
H. A. L. F. HOOGSTEDE ANd A. C. COPPER \\ From the Royal Netherlands Eye Hospital, Utrecht, The Netherlands
}

SUMMARY A 29-year-old man with a 7-year history of bilateral chronic uveitis developed subretinal neovascularisation in the macular area of his right eye. There was a history suggésting sarcoidosis. Long-term therapy with systemic corticosteroids decreased the activity of the uveitis, and the subretinal neovascularisation changed into a cystic scar. After the systemic corticosteroids were discontinued there was a recurrence of the uveitis but not of the subretinal neovascularisation.

Subretinal neovascularisation in young people has been reported in many diseases. In the absence of inflammatory signs in aqueous and vitreous or other specific ocular abnormalities it may be related to infection with Histoplasma capsulatum, ${ }^{1}$ but the same clinical picture has been observed in populations in which histoplasmosis is relatively rare. ${ }^{23}$

Some specific causes of uveitis can be complicated by subretinal neovascularisation, but only a few' reports have been delivered of subretinal neovascularisation in chronic aspecific uveitis. ${ }^{45}$

\section{Case report}

A man aged 29 years noted a sudden decrease of vision of his right eye in July 1979. Since 1972 he was known to be suffering from a bilateral chronic iridocyclitis without involvement of the posterior pole.

Medical examination in 1972 showed enlarged lymph nodes at the lung hila on chest $x$-ray. Because of a negative Mantoux reaction these changes were thought to be due to sarcoidosis. Seven months later the enlargement of the lymph nodes spontaneously regressed. No other abnormalities were found.

In July 1979 the corrected visuar acuity in the right eye was 0.16 and in the left eye 0.9 . Intraocular pressure measured by applanation tonometry was 13 $\mathrm{mmHg}$. The aqueous of both eyes showed flare and cells, and there were some old synechiae of the iris to the lens. The lenses were clear. The vitreous showed a

Correspondence to Dr H. A. L. F. Hoogstede, Koninklijk Nederlands Gasthuis voor Ooglijders, F.C. Dondersstraat 65, Utrecht, The Netherlands. small number of cells and somewhat more flare in the right than in the left eye.

Funduscopy of the right eye (Fig. 1) showed a subretinal haemorrhage and a grey local elevation of the retina in the macular area. In the periphery of the retina were some lesions of the pigment epithelium resembling atrophic scars. There were also some

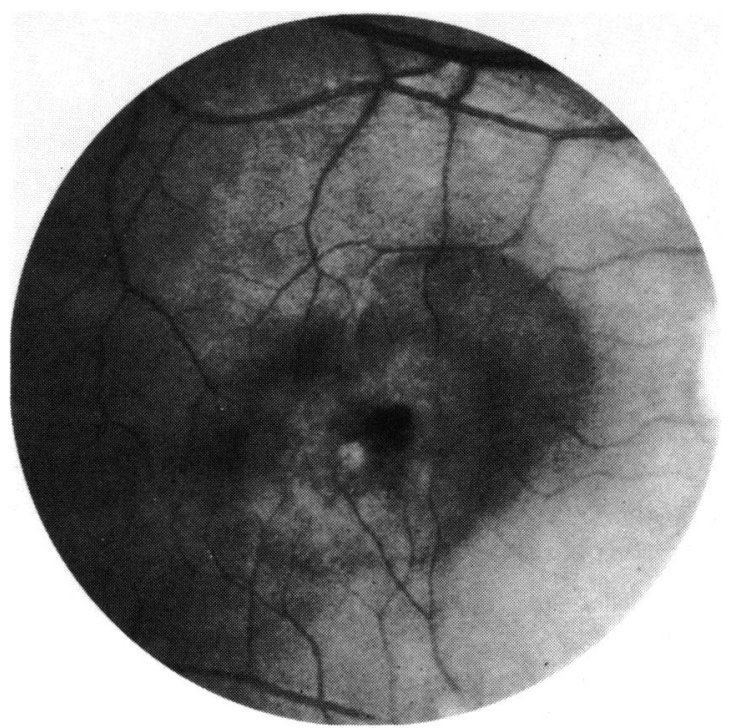

Fig. 1 Right eye fundus photograph, showing a foveal annular dark lesion, with a small subretinal haemorrhage. The detachment of the neuroepithelium covers the whole macular area. 


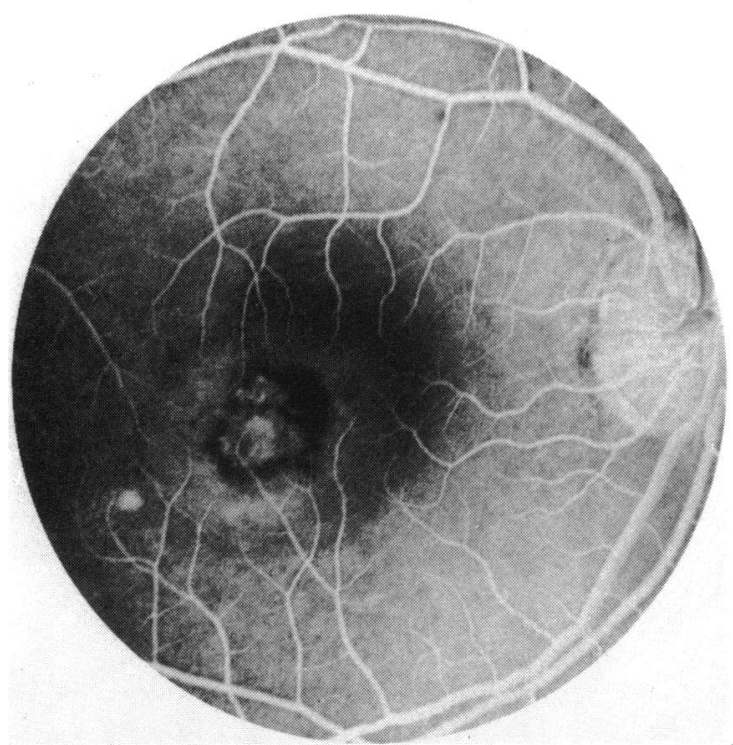

Fig. 2a

Figs. 2a,b,c Fluorescein angiogram of the right eye. (a) In the early arteriovenous phase a subretinal neovascular membrane; temporal to the macular area there is a small lesion in the pigment epithelium. (b) Leakage from the neovascular membrane in the venous phase. (c) $A$ pronounced leakage in the very late phase, and there is some leakage beneath the neuroepithelium detachment. The temporal pigment epithelial lesion shows some fluorescein staining.

round preretinal opacities in the vitreous. No signs of typical candle-wax dripping or vasculitis were present.

Funduscopy of the left eye showed a mild superficial macular oedema and also some peripheral scars in the epithelium. There were no local vitreous opacities or signs of vasculitis.

The fluorescein angiogram of the right fundus (Fig. 2) showed a pattern of active subretinal neovascularisation and a subretinal haemorrhage with a large overlying detachment of the neuroepithelium. In

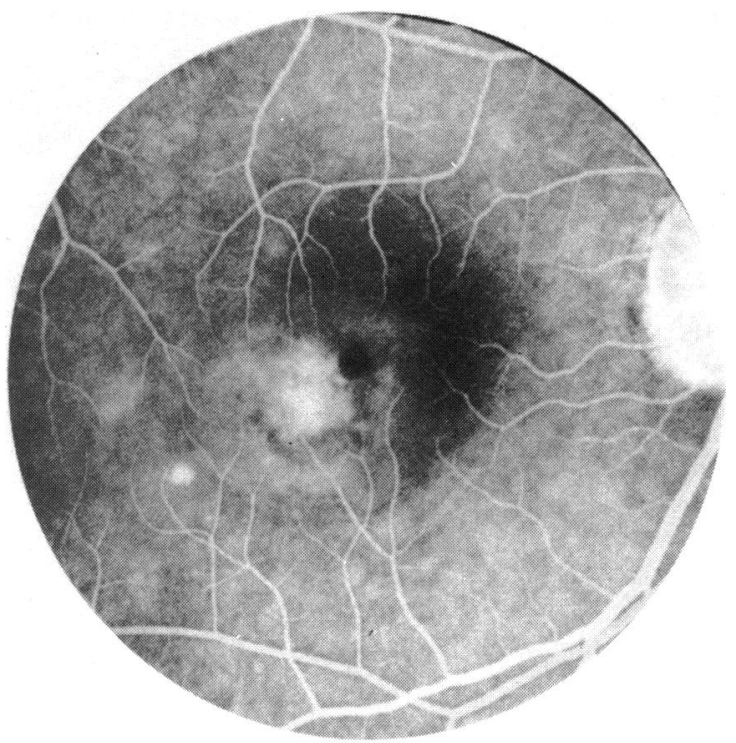

Fig. 2b

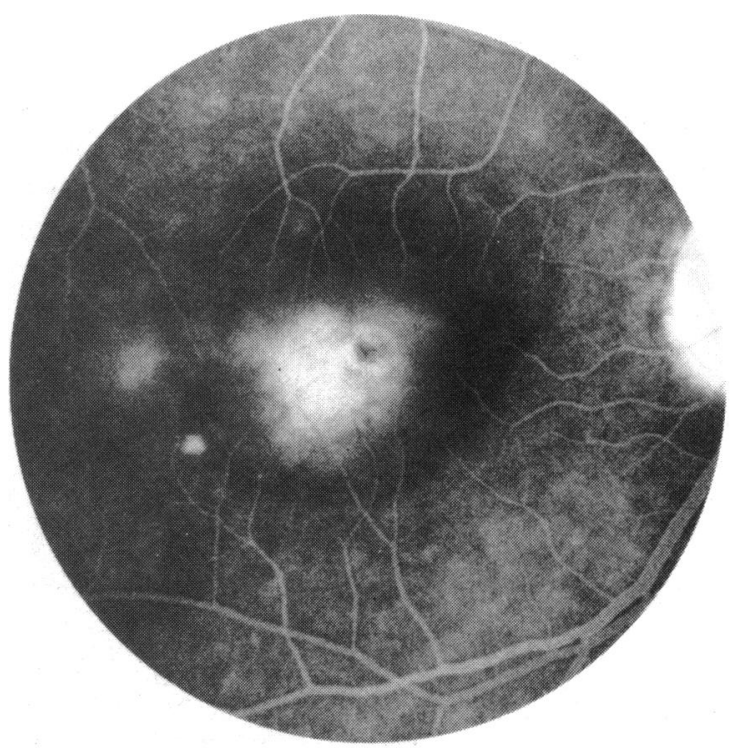

Fig. 2c

addition there was some leakage at the optic disc and a small lesion in the pigment epithelium next to the macula.

An angiogram of the left fundus (Fig. 3) showed a mild cystoid oedema in the macular area, with some leakage at the optic disc.

Renewed examination showed that the erythrocyte sedimentation rate, the serum electrolytes, and the haemoglobin level were all within normal limits. Liver and kidney functions were normal, as were the 


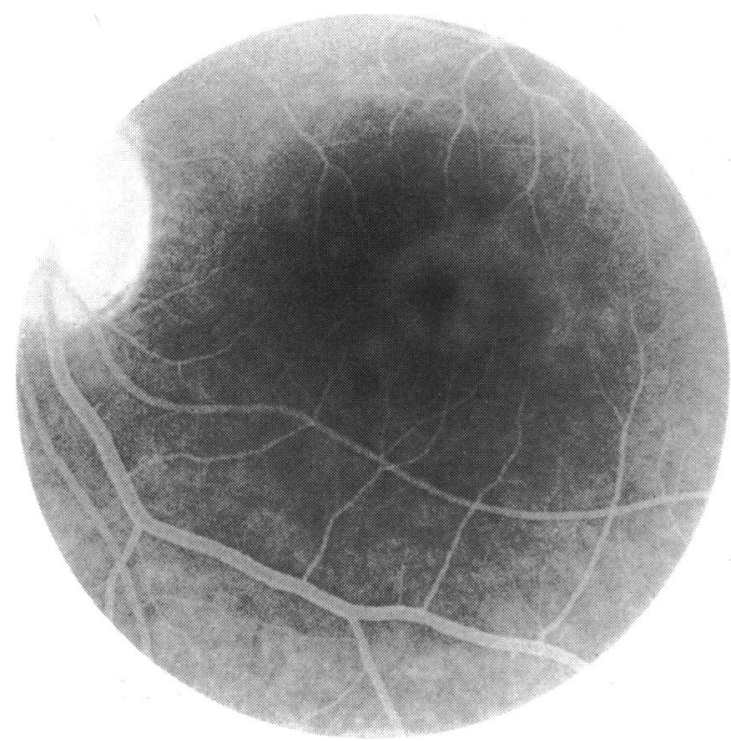

Fig. 3 Late phase of the angiogram of the left eye, showing a mild superficial leakage in the macular area and $a$ hyperfluorescence of the optic disc.

serum levels of calcium and phosphate. Tests for rheumatoid factor, antinuclear body, and toxoplasmosis serology were negative. The antistreptolysin titre was $275 \mathrm{U}$. $X$-rays of the chest, skull, and sinuses showed no abnormalities. The Mantoux

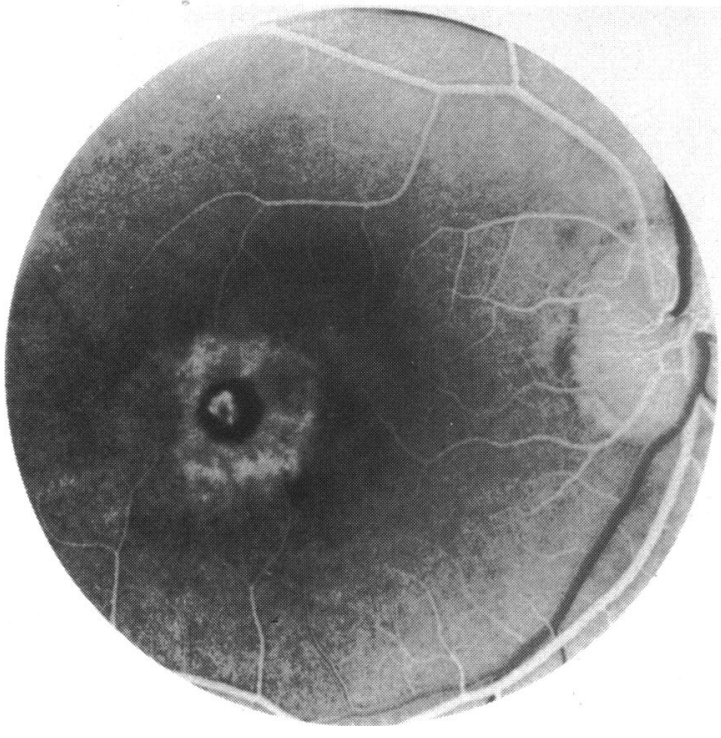

Fig. 5a

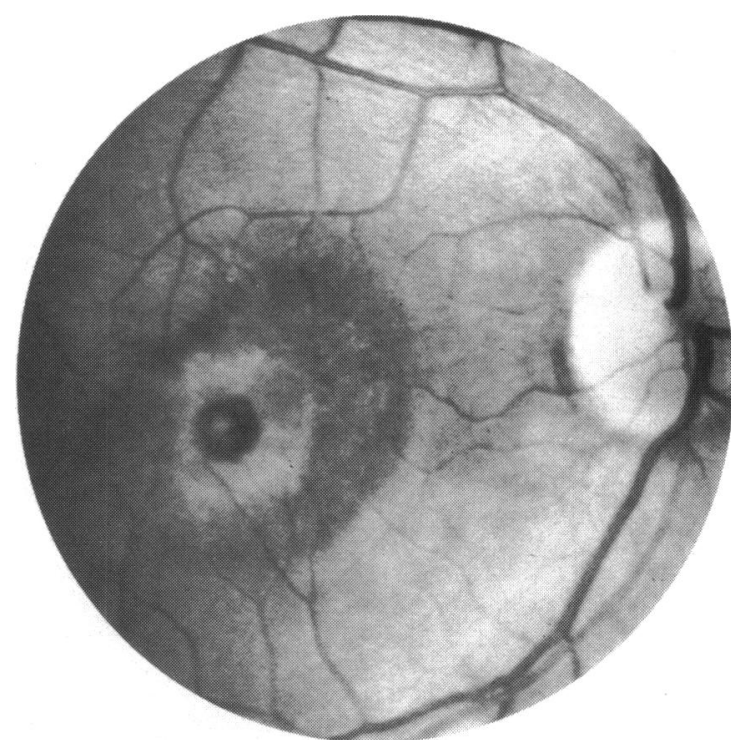

Fig. 4 Fundus photograph of the right eye showing a central dark cyst, surrounded by annular alterations of the pigment epithelium.

reaction and the skin test for histoplasmosis were negative.

Because of the signs of active uveitis, the superficial macular oedema, and the history of a possible sarcoidosis the patient was treated with systemic

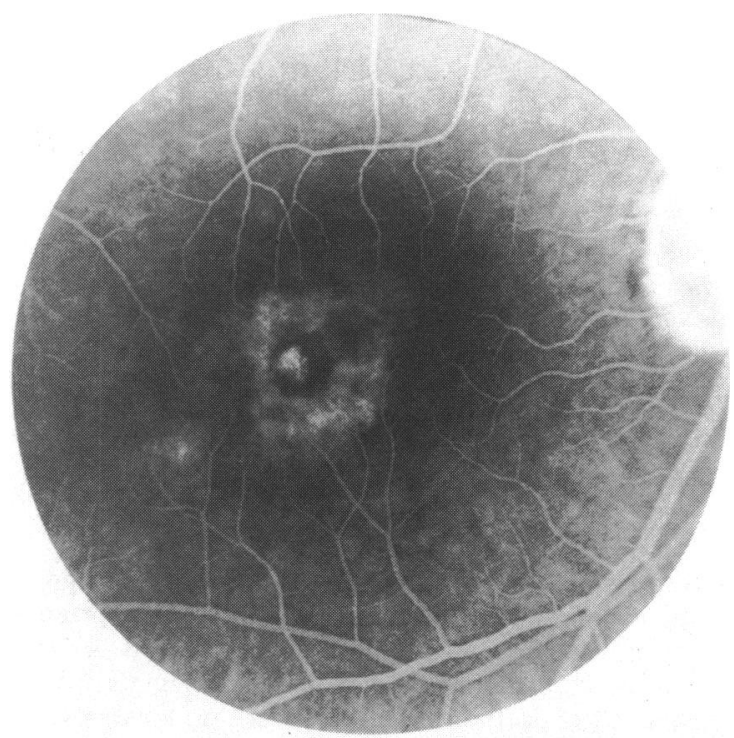

Fig. 5b

Figs. 5a,b Fluorescein angiogram of the right eye. (a) In the early arteriovenous phase a central hyperfluorescent lesion, surrounded by a hypofluorescent ring. There are no signs of active subretinal neovascularisation. The alterations at the pigment epithelium resemble window effects. (b) In the late phase no pronounced leakage in the macular area. 


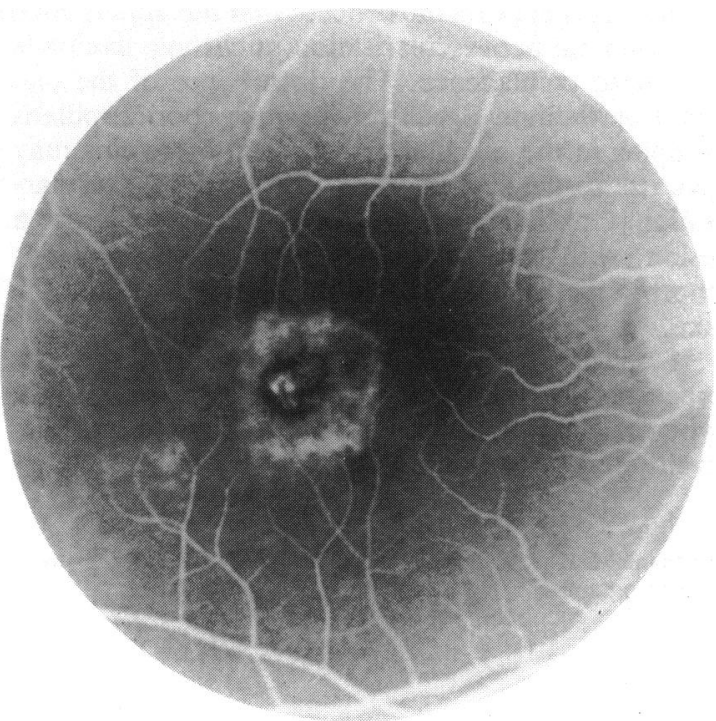

Fig. 6a

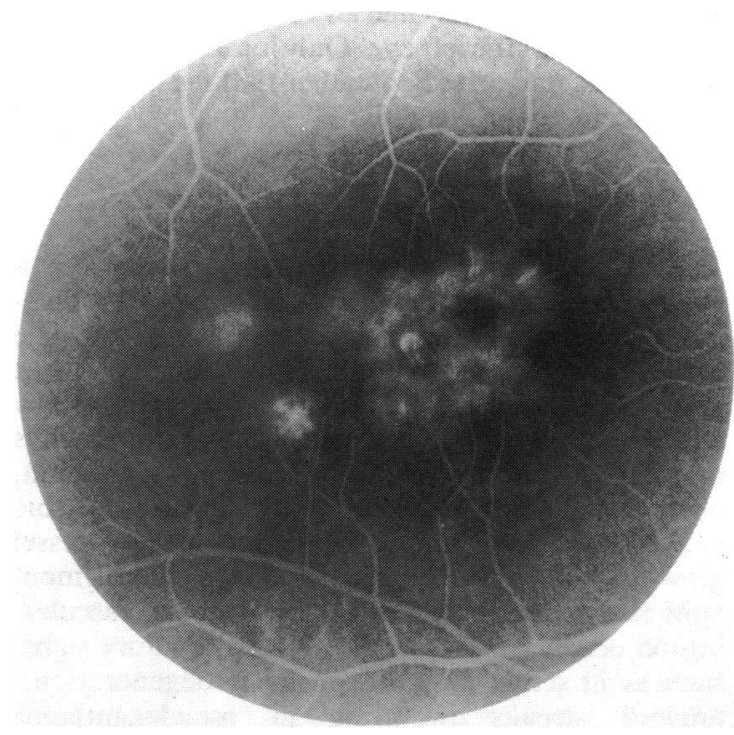

Fig. 6b

Figs. 6a,b Fluorescein angiogram of the right eye. (a) An early hyperfluorescence also in the central dark lesion. No signs of active subretinal neovascularisation. (b) In the late phase a merely superficial leakage over the macular area, but no leakage from deep layers, except from the lower temporal lesion located in the pigment epithelium.

corticosteroids, initially with $60 \mathrm{mg}$ prednisone dd. Within 8 weeks the dosage was lowered to $20 \mathrm{mg} \mathrm{d.d}$. and this level was maintained for some months.

Six months later the corrected visual acuity of the right eye had increased to 0.9 and of the left eye to $1 \cdot 2$. At that time there were no cells in the aqueous and only a mild flare in the vitreous. The macular lesion in the right eye (Fig. 4) had changed into a grey cystic scar without haemorrhage, and on the fluorescein angiogram (Fig. 5) the signs of activity of the subretinal neovascularisation had disappeared. In the left macular area a very mild superficial oedema remained. The systemic corticosteroids were gradually reduced and finally discontinued.

Ten months later there was an exacerbation of the uveitis in both eyes, with cells and flare in the aqueous and vitreous, and also a rise of intraocular pressure to $30 \mathrm{mmHg}$. The corrected visual acuity was 0.5 in the right eye and 0.8 in the left eye. The patient now refused treatment by systemic corticosteroids. For this reason only local corticosteroids in combination with antiglaucoma therapy were given.

A fluorescein angiogram of the right eye (Fig. 6) showed no activity of subretinal neovascularisation; only a cystic scar with surrounding pigment epithelium changes was visible. There was, however, some increase of the superficial macular oedema in both eyes (Fig. 7) and also some leakage at the optic discs.
Five months later the intraocular pressure was normal again, and there were minimal cells and flare in the aqueous and vitreous of both eyes. The macular lesion in the right eye did not change, and the

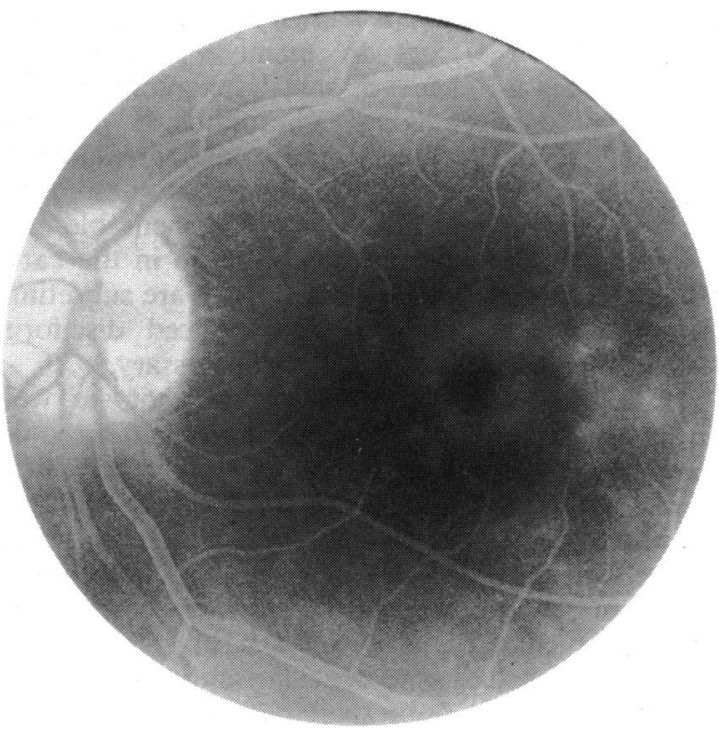

Fig. 7 Fluorescein angiogram of the left eye shows in the very late phase only a superficial macular leakage. The optic disc is hyperfluorescent. 
corrected visual acuity had now increased to 0.8 in the right and 0.9 in the left eye. Only local corticosteroids were given, and up to September 1981 the findings did not change.

\section{Discussion}

The occurrence of subretinal neovascularisation is probably related to changes in the pigment epitheliumBruch's membrane-choriocapillaris complex. The nature of the primary stimulating factors is not known. Ischaemia of the choriocapillaris (especially in the macular area), changes and breaks in Bruch's membrane, detachment of the pigment epithelium, haemorrhage, or other vasoproliferative factors could play a role in the stimulation of new vessel growth from the choroid into the subpigment epithelial space. Generally, subretinal neovascularisation occurs in the absence of inflammatory signs, such as in senile disciform macular degeneration, ${ }^{6}$ angioid streaks occurring in pseudoxanthoma elasticum, Ehlers-Danlos disease, Paget's disease of the bone, sickle-cell disease, the hereditary form of senile elastosis and acromegaly, ${ }^{67}$ myopia, ${ }^{8}$ vitteliform macular degeneration, ${ }^{9}$. hereditary drusen, ${ }^{10}$ Sorsby's pseudoinflammatory dystrophy, ${ }^{11}$ fundus flavimaculatus, ${ }^{12}$ traumatic choroidal rupture, ${ }^{6}$ focal macular choroidopathy, ${ }^{3}$ idiopathic subretinal neovascularisation, ${ }^{13}$ optic drusen, ${ }^{14}$ papilloedema by pseudotumor cerebri, ${ }^{15}$ following laser therapy, ${ }^{6}$ and in association with choroidal naevi and tumours. ${ }^{7}$

On the other hand it is known that subretinal neovascularisation occurs in mainly 'inflammatory' diseases such as Behçet's disease,${ }^{16}$ toxoplasmosis, ${ }^{17} 18$ toxocara, ${ }^{19}$ serpiginous choroiditis, ${ }^{20}$ chronic uveitis, ${ }^{45}$ Harada's disease, ${ }^{21}$ presumed sarcoidosis, ${ }^{22}$ rubella retinopathy, ${ }^{23}$ and presumed histoplasmosis. ${ }^{1}$

Fluorescein angiography is indispensable for detecting subretinal neovascularisation in the early stages. In the later stages, when there are subretinal haemorrhages or a more pronounced disciform lesion, an angiogram is not always necessary.

Subretinal neovascularisation is not a well recognised complication in chronic uveitis. This could be partly explained by the fact that some complications in chronic uveitis make it difficult to obtain a good fluorescein angiogram-for example, posterior synechiae of the iris, resulting in insufficient mydriasis, complicating cataract or vitreous opacities.

Fundus changes have often been described in sarcoidosis, including neovascularisation of the retina and optic disc. ${ }^{24}$ Subretinal neovascularisation in patients with presumed sarcoidosis was recently reported..$^{22}$ In our patient a history of sarcoidosis was suggested but was not proved histologically.
Our case supports the concept that the appearance of subretinal neovascularisation in chronic uveitis is not mere coincidence. The disturbance of the pigment epithelium-Bruch's membrane-choriocapillaris complex in the macular area caused by uveitis may give rise to the formation of subretinal neovascularisation. Ophthalmoscopically this can develop into a lesion with the aspect of the 'focal macular choroidopathy,' 'presumed histoplasmosis,' and the like.

If special attention is given to this aspectespecially to the minor forms-more cases of this type of subretinal neovascularisation in chronic uveitis may be found.

We thank Ms H. J. H. Schenk and Mr P. van Nigtevecht, who made the photographs.

\section{References}

1 Krill AE, Archer D. Choroidal neovascularisation in multifocal (presumed histoplasmine) choroiditis. Arch Ophthalmol 1970; 84: 595-604.

2 Braunstein RA, Rosen DA, Bird AC. Ocular histoplasmosis syndrome in the United Kingdom. Br J Ophthalmol 1974; 58: 893-8.

3 Craandijk A. Focal macular choroidopathy. Doc Ophthalmol 1979; 48: 1-103.

4 Schwartz PL, Gragoudas EW, Lapus JV. Peripapillary subretinal neovascularisation in chronic uveitis. Arch Ophthalmol 1978; 96: 836-8.

5 Augsburger JJ, Benson WE. Subretinal neovascularisation in chronic uveitis. Albrecht von Graefes Arch Klin Ophthalmol 1980; 215: 43-51.

6 Ryan SJR, Rainer NM, Maumenee AE. The disciform response: an historical perspective. Albrecht von Graefes Arch Klin Ophthalmol 1980; 215: 1-20.

7 Gass JDM. Pathogenesis of disciform detachment of the neuroepithelium. Am J Ophthalmol 1967; 63: 573-711.

8 Hotchkiss ML, Stuart LF. Pathologic myopia and choroidal neovascularisation. Am J.Ophthalmol 1981;91: 177-83.

9 Deutman AF. Unexpected findings in hereditary macular dystrophy. Doc Ophthalmol, Proceedings series, new developments in ophthalmology 1976; 7: 281-312.

10 Gass JDM. Stereoscopic Atlas of Macular Diseases. St Louis: Mosby, 1969: 13.

11 Ashton N, Sorsby A. Fundus dystrophy with unusual features: a historical study. Br J Ophthalmol 1951; 35: 751-64.

12 Klein R, Lewis RA, Meyers SM, Myers FL. Subretinal neovascularisation associated with fundus flavimaculatus. Arch Ophthalmol 1978; 96: 2054-67.

13 Cleasby GW. Idiopathic focal subretinal neovascularisation. Am J Ophthalmol 1976; 81: 590-6.

14 Yanuzzi LA, Gitter KA, Schatz H. The Macula. Baltimore: Williams and Wilkins, 1979: 288-9.

15 Jamison RR. Subretinal neovascularisation and papilledema associated with pseudotumor cerebri. Am J Ophthalmol 1978; 85: 78-81.

16 Michelson JB, Michelson PE, Chisari FV. Subretinal neovascular membrane and disciform scar in Behçet's disease. Am J Ophthalmol 1980; 90: 182-5.

17 Willerson D jr, Aaberg TM, Reeser F, Meredith TA. Unusual ocular presentation of acute toxoplasmosis. Br J Ophthalmol 1979; 61: 693-8. 
18 Fine SL, Owens SL, Haller JA, Knox DL, Patz A. Choroidal neovascularisation as late complication of ocular toxoplasmosis. Am J Ophthalmol 1980; 91: 318-22.

19 Gass JDM. Stereoscopic Atlas of Macular Diseases, Diagnosis and Treatment. St Louis: Mosby, 1977: 108.

20 Chisholm JH, Gass JDM, Hutton WL. The late stage of serpiginous (geographic) choroiditis. Am J Ophthalmol 1976; 82: 343-51.
21 Snyder DA, Tessler HH. Vogt-Koyanagi-Harada syndrome. Am J Ophthalmol 1980; 90: 69-75.

22 Gragoudas ES, Regan CDJ. Peripapillary subretinal neovascularisation in presumed sarcoidosis. Arch Ophthalmol 1981; 99: 1194-7.

23 Deutman AF, Sanderson Grizzard W. Rubella retinopathy and subretinal neovascularization. Am J Ophthalmol 1978; 85: 82-7.

24 Spalton DJ, Sanders MD. Fundus changes in histologically confirmed sarcoidosis. Br J Ophthalmol 1981; 65: 348-58. 